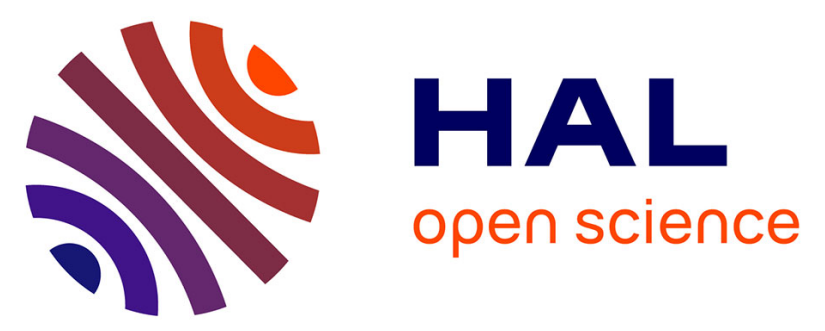

\title{
Determination of deviatoric elastic strain and lattice orientation by applying digital image correlation to Laue microdiffraction images: The enhanced Laue-DIC method
}

F. G. Zhang, Olivier Castelnau, Michel Bornert, Johann Petit, Jean Baptiste Marijon, Emeric Plancher

\section{To cite this version:}

F. G. Zhang, Olivier Castelnau, Michel Bornert, Johann Petit, Jean Baptiste Marijon, et al.. Determination of deviatoric elastic strain and lattice orientation by applying digital image correlation to Laue microdiffraction images: The enhanced Laue-DIC method. Journal of Applied Crystallography, 2015, 48 (6), pp.1805-1817. 10.1107/S1600576715018397 . hal-02141076

\author{
HAL Id: hal-02141076 \\ https://hal.science/hal-02141076
}

Submitted on 27 May 2019

HAL is a multi-disciplinary open access archive for the deposit and dissemination of scientific research documents, whether they are published or not. The documents may come from teaching and research institutions in France or abroad, or from public or private research centers.
L'archive ouverte pluridisciplinaire HAL, est destinée au dépôt et à la diffusion de documents scientifiques de niveau recherche, publiés ou non, émanant des établissements d'enseignement et de recherche français ou étrangers, des laboratoires publics ou privés. 
Edited by A. Borbély, Ecole National Supérieure des Mines, Saint-Etienne, France

Keywords: deviatoric elastic strain; lattice orientation; digital image correlation; Laue microdiffraction.
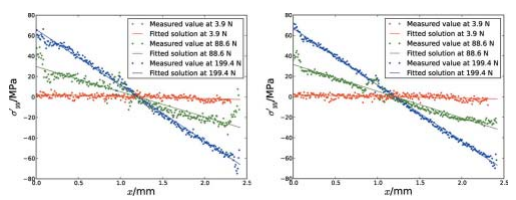

(C) 2015 International Union of Crystallography

\section{Determination of deviatoric elastic strain and lattice orientation by applying digital image correlation to Laue microdiffraction images: the enhanced Laue-DIC method}

\author{
F. G. Zhang, ${ }^{a}$ O. Castelnau, ${ }^{\mathrm{a} *}$ M. Bornert, ${ }^{\mathrm{b}}$ J. Petit, ${ }^{\mathrm{c}}$ J. B. Marijon ${ }^{\mathrm{a}}$ and E. Plancher ${ }^{\mathrm{a}}$ \\ aPIMM CNRS UMR 8006, Arts et Métiers ParisTech, 151 Boulevard de l'Hôpital, 75013 Paris, France, 'baboratoire \\ NAVIER, ENPC/IFSTTAR/CNRS UMR 8205, Université Paris-Est, 77455, Marne-la-Vallée, France, and 'LEME CNRS EA \\ 4416, Université Paris-Ouest, 50 Rue de Sèvres, 92410, Ville d'Avray, France. *Correspondence e-mail: \\ olivier.castelnau@ensam.eu
}

A new method of determining the deviatoric elastic strain and lattice orientation from Laue microdiffraction images is presented. Standard data treatment methods can suffer from the difficulty of precisely pinpointing the positions of diffraction peaks on two-dimensional Laue images. In a previous article, digital image correlation (DIC) was introduced for the treatment of Laue images, leading to the so-called Laue-DIC method. This performed better than the standard method in terms of the deviatoric elastic strain increment and relative rotation from one lattice to another, particularly when the shape of the Laue spots departs from regular ellipsoids. The present work intends to push forward the Laue-DIC method, aiming to determine the deviatoric elastic strain and lattice orientation, as well as the calibration parameters. The performance of this new method, named enhanced Laue-DIC, is assessed by modeling the spot displacements and accounting for random fluctuations relevant for typical experimental conditions. When the enhanced Laue-DIC method is applied to the case of an in situ deformed Si crystal, the obtained standard deviation of local stress is of the order of 1-2 MPa, while the calibration parameters are optimized to high accuracy.

\section{Introduction}

$\mathrm{X}$-ray Laue microdiffraction is a powerful technique to investigate intragranular orientation and elastic deformation at a fine scale thanks to its sub-micrometre focal size. At present, beamline BM32 at the European Synchrotron Radiation Facility (ESRF) can provide a beam of $\sim 0.5 \times$ $0.7 \mu \mathrm{m}^{2}$, with a spectral bandwidth of 5-25 keV (Ulrich et al., 2011; Robach et al., 2014). In contrast to other X-ray diffraction techniques using a monochromatic beam, for example the $\sin ^{2} \psi$ method (Macherauch, 1966), Laue microdiffraction, using a polychromatic beam, requires no rotation of the sample or detector. This is an important point when one wants to reach submicrometre spatial resolution. To orient a single crystal in a monochromatic beam, three sample rotations are needed. Although recent air-bearing systems allow the sphere of confusion of one rotation to be smaller than a fraction of a micrometre (see e.g. Xu et al., 2014; Waltersperger et al., 2015), the authors are not aware of three-axis goniometers with a sphere of confusion smaller than a few micrometres, i.e. an order of magnitude larger than the actual beam size. Any sample rotation would then move the measurement point out of the beam (Castelnau et al., 2001). Rotation would also lead to the ambiguity of the illuminated volume, owing to the beam 
penetration, an ambiguity not present with Laue microdiffraction.

Given these advantages, Laue microdiffraction finds its widespread application in measuring strain/stress at the submicrometre scale, such as in thin films (Tamura et al., 2003), in metallic micropillars (Maaß et al., 2009), at bi-crystal boundaries (Ohashi et al., 2009) and at tri-crystal boundaries (Daveau, 2012). Although high-resolution electron backscattered diffraction (HR-EBSD) allows for even finer spatial resolution ( $\sim 10 \mathrm{~nm}$ ) (Villert et al., 2009), Laue microdiffraction allows the additional capability of through-thickness spatial resolution of elastic strain using the differential aperture X-ray microscopy method (Yang et al., 2004). A comparison between HR-EBSD and Laue microdiffraction in plastically deformed single crystals can be found in the article by Plancher et al. (2015).

Laue diffraction patterns are usually recorded on an area detector, and codes for deducing lattice orientation and elastic strain from recorded images are already available, such as XMAS (Tamura, 2014) and LaueTools (http://sourceforge.net/ projects/lauetools/). The method used in these codes is largely based on the algorithm presented by Chung \& Ice (1999) and allows for a rapid indexing of Laue spots and the estimation of the lattice matrix. Conventionally, peak positions are pinpointed by fitting spots with some analytical functions. However, the commonly used analytical functions, usually Gaussian or Lorentz functions, implicitly assume an elliptical spot shape, limiting potential applications. Indeed, there are many situations for which spots depart from ellipticity, for example when the crystal has undergone plastic strain and contains geometrically necessary dislocations (see, for instance, Ohashi et al., 2009), or when a strain gradient is present within a pure crystals and produces mirage spots due to dynamic effects (Yan \& Noyan, 2006).

Another difficulty with the standard method is that the calibration of the experimental setup must be given very accurately, as it is necessary to transform the rectangular coordinates of the peak position into angular coordinates. Any error in the calibration will ultimately contribute to the error of elastic strain assessment (Hofmann et al., 2011).

This paper is a continuation of the work of Petit et al. (2015), in which a method called Laue-DIC (referred to as original Laue-DIC hereinafter) was proposed: unlike the standard method, the peak positions were no longer fitted by any analytical function, but their displacements from one Laue image to another were measured by a well established technique - digital image correlation (referred to as DIC hereinafter) - in light of its high sub-pixel accuracy (Bornert et al., 2009; Amiot et al., 2013). It was shown that Laue-DIC reached an accuracy of the order of $10^{-5}$ for measuring elastic strain increment (more specifically, the deviatoric components of elastic strain). In this paper, we present an enhanced version of Laue-DIC (referred to as enhanced Laue-DIC hereinafter). By using the term 'enhanced', we mean that the enhanced version is no longer limited to seeking elastic strain increments between two lattices, as in the original version, but can be used to find the deviatoric lattice matrices and calibration para- meters as an ensemble. For a full determination of all components of lattice matrices, and in particular their traces, some additional information is required, such as photon energies (Robach et al., 2011).

The paper is structured as follows. We will introduce the context and the standard procedure in $\$ 2$. Then, we will briefly review original Laue-DIC and present its enhanced version in $\S 3$. The efficiency of enhanced Laue-DIC will be assessed by numerical tests in $\$ 4$. Finally, in $\$ 5$, we will compare the performance of the standard method, original Laue-DIC and enhanced Laue-DIC through the processing of experimental images, and comment on their differences.

\section{Context and brief review of the standard procedure}

In Laue microdiffraction experiments, the distribution of Laue spots on an area detector is determined by two factors: the lattice that diffracts the X-rays, and the setup of the area detector. In this paper, the term 'configuration' is used to refer to both of these factors combined. First, let us introduce the setup of the area detector in Laue microdiffraction.

The relative spatial relation between the incident beam and the area detector can be characterized by two angles. Let us first establish an absolute coordinate system (referred to as $\mathfrak{R}$ ) upon which our calculation will be based (see Fig. 1). The $y$ axis of $\mathfrak{R}$ is along the incident beam. The origin $\mathrm{O}$ is the illuminated point on the sample surface, and $\mathrm{P}$ is the projection of $\mathrm{O}$ onto the area detector, which is modeled as a plane. Then the $x$ axis is defined as

$$
\mathbf{e}_{x} \doteq \frac{\mathbf{e}_{y} \times \mathbf{O P}}{\left\|\mathbf{e}_{y} \times \mathbf{O P}\right\|},
$$

and the $z$ axis is defined as

$$
\mathbf{e}_{z} \doteq \mathbf{e}_{x} \times \mathbf{e}_{y} .
$$

The setup of the area detector can be characterized by five parameters: $x_{\mathrm{c}}, y_{\mathrm{c}}, \beta, \gamma$ and $d$ (Robach et al., 2011), where $d$ is the distance between the illuminated site and the area detector, i.e. $d=\|\mathbf{O P}\|, \beta$ is the angle between the $z$ axis and $\mathbf{O P}, \gamma$ is the rotation angle of the area detector around $\mathbf{O P}$, which determines the direction of rows and columns of pixels on the detector with respect to the overall frame, and $x_{\mathrm{c}}, y_{\mathrm{c}}$ are

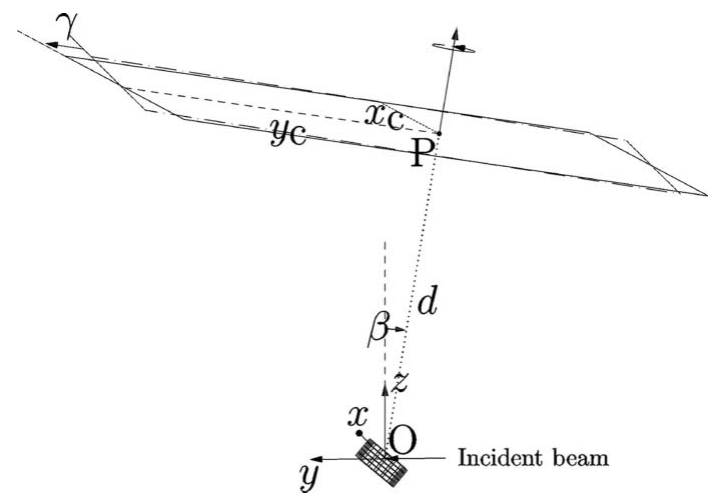

Figure 1

Calibration parameters of the setup of the area detector. 
the distances of $\mathrm{P}$ from the two perpendicular edges of the area detector. Pixels on the detector are assumed to be arranged in a perfect square array with a known step.

With these calibration parameters, the rectangular pixel coordinates of a peak on the area detector can be readily transformed into its angular coordinates in $\Re$. These parameters are usually obtained using a well known stress-free single crystal (e.g. Ge) by adjusting the calibration parameters to minimize the discrepancy between the simulated and measured peak positions (Robach et al., 2011). Then, the experimenter carefully handles the sample to keep the calibration parameters constant when shifting the region of interest of the sample into the beam, so that the illumination point remains at the same position. Once peaks are pinpointed and transformed into their angular positions, the indexing of peaks and the lattice matrix of the illuminated volume can be determined (Chung \& Ice, 1999).

However, the procedure described above (denoted hereafter as the 'standard method') is prone to two sources of uncertainty (Poshadel et al., 2012):

(a) Peak positions. Locating the peak position is usually accomplished by fitting the gray level intensity distribution on the Laue image with an analytical function including a small number of parameters (such as a two-dimensional Gaussian function). Such a procedure is not appropriate when the spots exhibit irregular shapes. Examples include diffraction from plastically deformed crystals (Barabash et al., 2001) and from a bent perfect crystal (Yan \& Noyan, 2006).

(b) Calibration parameters. The calibration is performed with a crystal with known lattice parameters as mentioned before. When the illumination site is shifted to the region of interest, small perturbations of calibration parameters are inevitable. The calibration may also be modified by a slight beam displacement (micrometre range), which may be caused by many possible factors. Moreover, the difference in penetration depth between the calibration crystal and the sample will contribute to the uncertainty of the results as well (Hofmann et al., 2011).

\section{Enhanced Laue-DIC}

\subsection{Brief review of original Laue-DIC}

In order to tackle some of the aforementioned sources of uncertainty, Petit et al. (2015) proposed the original Laue-DIC method, in which one no longer needs to determine peak positions very accurately (e.g. by analytical fitting), but rather the accurate peak displacement between two images (measured by DIC) is of interest (see Fig. 2). This preference to measure displacements by DIC is supported by the following reasons:

(1) DIC measures peak displacements by taking advantage of the resemblance of spots in two diffraction images. The spots' resemblance can be easily guaranteed as long as the substructures within the diffraction volumes of the two images differ little, while analytical fitting requires the regularity of spots, which is more difficult to guarantee.
(2) The displacements obtained by DIC have better accuracy than the absolute positions of peaks obtained by analytical fitting. It is reported that the accuracy of DIC can be of the order of a few hundredths of a pixel or even less (Bornert et al., 2009; Amiot et al., 2013) when applied on a pair of images with good quality, while the uncertainty of the peak position obtained by analytical fitting is usually 0.1 pixel, even for spots of good quality (Poshadel et al., 2012).

In the original Laue-DIC procedure, two images are compared using DIC. The first image refers to a reference mechanical configuration, and the second image to the current configuration. The method then consists of the following steps (Petit et al., 2015):

(1) First, the LaueTools software (for example) is used to obtain a guess value of the crystal orientation and the deviatoric elastic strain of the initial configuration.

(2) Next, DIC is used to measure the spot motion field on the area detector between the two images (or equivalently, between the two mechanical configurations).

(3) Finally, a cost function minimization is run to evaluate the deviatoric components of the incremental elastic strain and relative rotation of the lattice between the two configurations that best match the measured evolution of the Laue pattern.

Whilst original Laue-DIC has been shown to improve the resolution of the measurement of the elastic strain increment (hence stress increment) between two configurations, this paper will extend Laue-DIC to the evaluation not only of the deviatoric components but also of lattice matrices and the calibration parameters, leading to an enhanced version of Laue-DIC. Before presenting enhanced Laue-DIC, let us first briefly introduce the principle of DIC.

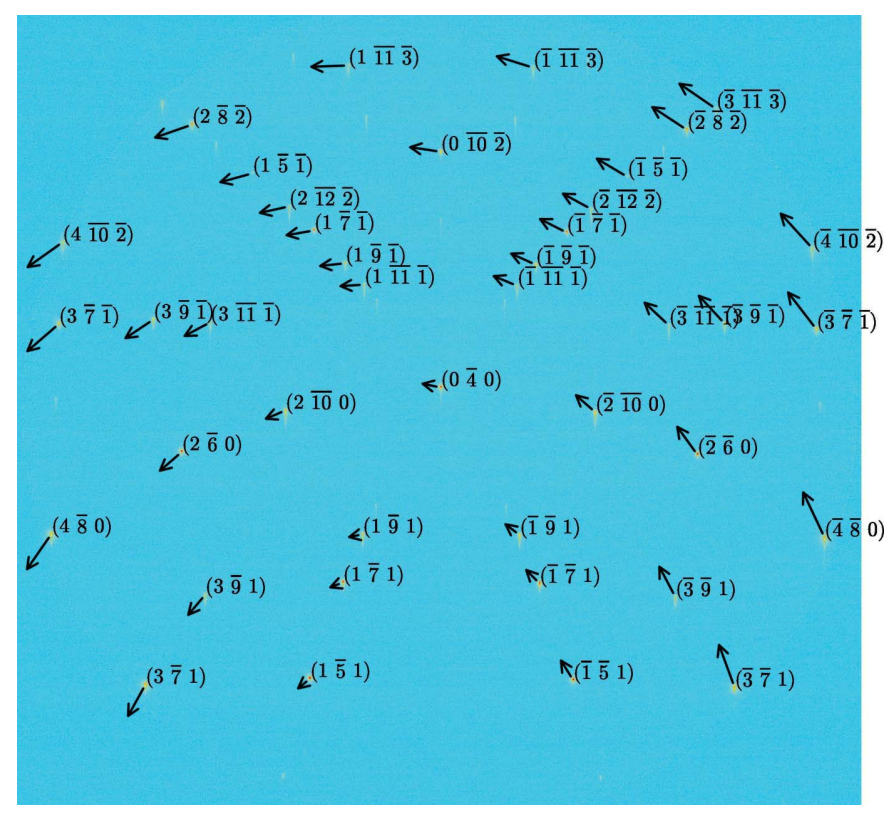

Figure 2

Simulated displacements of Laue spots. Laue spots were collected from an Si crystal undergoing bending and labeled with their Miller indexes. Their displacements on the detector screen are as depicted by the arrows (magnified by a factor 50). 


\subsection{DIC}

DIC is an optical technique to acquire the displacement/ strain field of an object's surface. Its history can be traced to the beginning of the 1980s (Peters \& Ranson, 1982). The practitioner of DIC needs to designate a set of sub-images as a 'zone of interest' (ZOI) on the acquired image of the object's surface. Then the aim of DIC is to seek the displacement field within the ZOI by matching images captured at intervals of the object's motion. The motion of the ZOI is characterized by a polynomial expression (the so-called shape function). The simplest form of shape function is a zero-order polynomial associated with rigid translation. DIC does the matching by minimizing the so-called correlation coefficient, a scalar quantification of image resemblance. Various forms of correlation coefficient are available (Amiot et al., 2013). The simplest form of correlation coefficient is the sum of squared difference (SSD):

$$
C_{\mathrm{SSD}} \doteq \sum\left(f_{i}-g_{i}\right)^{2},
$$

where $f_{i}$ is the gray level at the $i$ th pixel of the so-called reference image, while $g_{i}$ is the interpolated gray level in the so-called deformed image, at the position associated with this pixel according to the shape function.

In this work, DIC was performed using the in-house software $C M V$ (Doumalin \& Bornert, 2000; Bornert et al., 2010). The $C M V$ code adopts the zero-mean normalized crosscorrelation (ZNCC) coefficient:

$$
C_{\mathrm{ZNCC}} \doteq 1-\frac{\sum\left(f_{i}-\bar{f}\right)\left(g_{i}-\bar{g}\right)}{\left[\sum\left(f_{i}-\bar{f}\right)^{2} \sum\left(g_{i}-\bar{g}\right)^{2}\right]^{1 / 2}},
$$

where $\bar{f}$ and $\bar{g}$ are the averages of $f_{i}$ and $g_{i}$, respectively. Though ZNCC is much more complex than SSD, it is insensitive to the offset and scale changes in the gray level of images and gives the best results compared to other correlation coefficients in terms of displacement measurement (Tong, 2005). This property is especially useful when the illumination conditions cannot guarantee the strict conservation of gray levels, as for instance in scanning electron microscopy images (Doumalin \& Bornert, 2000).

A great benefit of DIC for Laue images lies in the fact that the detection of spot motion is basically insensitive to the spot shape. Therefore, we anticipate that the technique presented below should work for crystals having undergone plastic strain and containing geometrically necessary dislocations, for which spot shapes are complex; the important point is that spot shapes do not evolve during the considered deformation increment. Wang et al. (2009) derived complex formulas to estimate the errors of DIC given the gray level distribution and noise of images. According to these formulas, the error of DIC could be of the order of $10^{-3}$ pixels for our images.

\subsection{Principle of enhanced Laue-DIC}

In the following, Einstein's summation convention (implicit summation over repeated indices) will be employed for the sake of brevity. The lattice cell of the crystal of interest is defined by its three lattice vectors, $\mathbf{a}, \mathbf{b}$ and $\mathbf{c}$. These vectors are grouped into a lattice matrix, whose columns correspond to the lattice vectors:

$$
\mathbf{l}=[\mathbf{a}, \mathbf{b}, \mathbf{c}] .
$$

With the lattice matrix $\mathbf{l}$, we can define its reciprocal lattice matrix $\mathbf{I}^{*}=\left[\mathbf{a}^{*}, \mathbf{b}^{*}, \mathbf{c}^{*}\right]$, whose columns are the reciprocal lattice vectors of $\mathbf{a}, \mathbf{b}$ and $\mathbf{c}$ :

$$
\mathbf{a}^{*} \doteq \frac{\mathbf{b} \times \mathbf{c}}{\mathbf{a} \cdot(\mathbf{b} \times \mathbf{c})}, \quad \mathbf{b}^{*} \doteq \frac{\mathbf{c} \times \mathbf{a}}{\mathbf{a} \cdot(\mathbf{b} \times \mathbf{c})}, \quad \mathbf{c}^{*} \doteq \frac{\mathbf{a} \times \mathbf{b}}{\mathbf{a} \cdot(\mathbf{b} \times \mathbf{c})} .
$$

In Laue microdiffraction, we do not directly obtain the lattice matrix l. Rather, we obtain its reciprocal lattice matrix $\mathbf{I}^{*}=\mathbf{I}^{-\mathrm{T}}$ (the superscript $-\mathrm{T}$ denotes the transposed inverse matrix), since the diffraction condition can be more conveniently expressed in reciprocal space:

$$
\mathbf{k}^{\mathrm{f}}-\mathbf{k}^{\mathrm{i}}=\mathbf{I}^{*} \cdot \mathbf{h},
$$

where $\mathbf{k}^{\mathrm{f}}$ and $\mathbf{k}^{\mathrm{i}}$ are the wavevectors of the diffracted beam and the incident beam, respectively, and $\mathbf{h}=[h, k, l]^{\mathrm{T}}$ is the index of diffraction. Note that $\mathbf{k}^{\mathrm{f}}$ and $\mathbf{k}^{\mathrm{i}}$ are of the same magnitude, i.e. $\left\|\mathbf{k}^{\mathrm{f}}\right\|=\left\|\mathbf{k}^{\mathrm{i}}\right\|=1 / \lambda$, where $\lambda$ is the wavelength. Therefore, the unit vectors of $\mathbf{k}^{\mathrm{f}}$ and $\mathbf{k}^{\mathrm{i}}$, denoted $\hat{\mathbf{k}}^{\mathrm{f}}$ and $\hat{\mathbf{k}}^{\mathrm{i}}$, are linked by the following relation:

$$
\hat{\mathbf{k}}^{\mathrm{f}}-\hat{\mathbf{k}}^{\mathrm{i}}=\left(\mathbf{k}^{\mathrm{f}}-\mathbf{k}^{\mathrm{i}}\right) /(1 / \lambda)=\lambda \mathbf{I}^{*} \cdot \mathbf{h}
$$

or

$$
\hat{\mathbf{k}}^{\mathrm{f}}=\hat{\mathbf{k}}^{\mathrm{i}}+\lambda \mathbf{I}^{*} \cdot \mathbf{h} .
$$

Taking the squared norm on both sides of equation (9) gives

$$
1=1+2 \lambda \hat{\mathbf{k}}^{\mathrm{i}} \cdot\left(\mathbf{I}^{*} \cdot \mathbf{h}\right)+\lambda^{2}\left\|\mathbf{I}^{*} \cdot \mathbf{h}\right\|^{2} .
$$

Hence,

$$
\lambda=-2 \frac{\hat{\mathbf{k}}^{\mathrm{i}} \cdot\left(\mathbf{l}^{*} \cdot \mathbf{h}\right)}{\left\|\mathbf{l}^{*} \cdot \mathbf{h}\right\|^{2}} .
$$

Substituting equation (11) into equation (9) leads to

$$
\hat{\mathbf{k}}^{\mathrm{f}}=\hat{\mathbf{k}}^{\mathrm{i}}-2 \frac{\left[\hat{\mathbf{k}}^{\mathrm{i}} \cdot\left(\mathbf{I}^{*} \cdot \mathbf{h}\right)\right]}{\left\|\mathbf{I}^{*} \cdot \mathbf{h}\right\|^{2}} \mathbf{I}^{*} \cdot \mathbf{h} .
$$

Note that in $\mathfrak{R}$ the incident beam is collimated with the $y$ axis. Therefore the components of $\hat{\mathbf{k}}^{\mathrm{i}}$ in $\Re$ are constantly $\hat{\boldsymbol{k}}_{i}^{\mathrm{i}} \equiv \delta_{2 i}$ ( $\delta_{i j}$ is the Kronecker delta), and the components of $\hat{\mathbf{k}}^{\mathrm{f}}$ can be written as

$$
\hat{k}_{i}^{\mathrm{f}}=\frac{\left\|\mathbf{I}^{*} \cdot \mathbf{h}\right\|^{2} \delta_{2 i}-2 l_{2 j}^{*} h_{j} l_{i r}^{*} h_{r}}{\left\|\mathbf{I}^{*} \cdot \mathbf{h}\right\|^{2}} .
$$

The interception of $\hat{\mathbf{k}}^{\mathrm{f}}$ by the area detector forms a Bragg peak. The coordinates of the peak in the detector reference frame, denoted $(x, y)$, have the following relation with $\hat{\mathbf{k}}^{\mathrm{f}}$ according to Fig. 1:

$$
\frac{\hat{k}_{i}^{\mathrm{f}} g_{1 i}}{x-x_{\mathrm{c}}}=\frac{\hat{k}_{i}^{\mathrm{f}} g_{2 i}}{y-y_{\mathrm{c}}}=\frac{\hat{k}_{i}^{\mathrm{f}} g_{3 i}}{d},
$$

where $g_{i j}$ are the components of $\mathbf{g}$, the matrix transforming the coordinates in $\Re$ into the detector coordinate system: 
Table 1

Symbols for quantities in the reference and current configurations.

\begin{tabular}{lllllllll}
\hline Reference configuration & $D$ & $X_{\mathrm{c}}$ & $Y_{\mathrm{c}}$ & $\mathrm{B}$ & $\Gamma$ & $\mathbf{L}^{*}$ & $\mathbf{G}$ & $\mathbf{S}$
\end{tabular}

$\begin{array}{lllllllll}\text { Current configuration } & d & x_{\mathrm{c}} & y_{\mathrm{c}} & \beta & \gamma & \mathbf{l}^{*} & \mathbf{g} & \mathbf{s}\end{array}$

$$
\mathbf{g}=\left[\begin{array}{ccc}
\cos \gamma & -\cos \beta \sin \gamma & \sin \beta \sin \gamma \\
\sin \gamma & \cos \beta \cos \gamma & -\sin \beta \cos \gamma \\
0 & \sin \beta & \cos \beta
\end{array}\right] .
$$

Then $(x, y)$ can be expressed as

$$
x=d \frac{\hat{k}_{i}^{\mathrm{f}} g_{1 i}}{\hat{k}_{i}^{\mathrm{f}} g_{3 i}}+x_{\mathrm{c}}, \quad y=d \frac{\hat{k}_{i}^{\mathrm{f}} g_{2 i}}{\hat{k}_{i}^{\mathrm{f}} g_{3 i}}+y_{\mathrm{c}} .
$$

Because the expressions of $\hat{k}_{1}^{\mathrm{f}}, \hat{k}_{2}^{\mathrm{f}}$ and $\hat{k}_{3}^{\mathrm{f}}$ in equation (13) have the same denominator $\left\|\mathbf{I}^{*} \cdot \mathbf{h}\right\|^{2}$, we can define a scaled version of $\mathbf{k}^{\mathrm{f}}$ :

$$
\mathbf{s} \doteq\left\|\mathbf{I}^{*} \cdot \mathbf{h}\right\|^{2} \hat{\mathbf{k}}^{\mathrm{f}}=\left\|\mathbf{I}^{*} \cdot \mathbf{h}\right\|^{2} \hat{\mathbf{k}}^{\mathrm{i}}-2\left[\hat{\mathbf{k}}^{\mathrm{i}} \cdot\left(\mathbf{I}^{*} \cdot \mathbf{h}\right)\right] \mathbf{I}^{*} \cdot \mathbf{h} .
$$

Its components in $\mathfrak{R}$ are

$$
s_{i}=\left\|\mathbf{I}^{*} \cdot \mathbf{h}\right\|^{2} \delta_{2 i}-2 l_{2 j}^{*} h_{j} l_{i r}^{*} h_{r}=l_{p m}^{*} h_{m} l_{p n}^{*} h_{n} \delta_{2 i}-2 l_{2 j}^{*} h_{j} l_{i r}^{*} h_{r} .
$$

The coordinates of the diffracted peak on the area detector can be expressed as

$$
x=d \frac{s_{i} g_{1 i}}{s_{i} g_{3 i}}+x_{\mathrm{c}}, \quad y=d \frac{s_{i} g_{2 i}}{s_{i} g_{3 i}}+y_{\mathrm{c}} .
$$

In enhanced Laue-DIC, the obtained lattice matrices and calibration parameters in two configurations are considered instead of the increment of elastic strain and lattice rotation between two configurations as in original Laue-DIC. Here, the calibration parameters and lattice matrix of the reference configuration are denoted by uppercase letters, and those of the current configuration by lowercase letters (see Table 1). Note that in enhanced Laue-DIC the designation of reference or current configuration can be arbitrary, while in original Laue-DIC it is necessary to denote as the reference configuration the one whose lattice matrices and calibration parameters are known accurately in advance, otherwise only the increments of deviatoric elastic strain or stress between two configurations can be evaluated.

The displacement of a Bragg peak between the reference and the current configurations can be expressed as

$\Delta x=d \frac{s_{i} g_{1 i}}{s_{i} g_{3 i}}-D \frac{S_{i} G_{1 i}}{S_{i} G_{3 i}}+\Delta x_{\mathrm{c}}, \quad \Delta y=d \frac{s_{i} g_{2 i}}{s_{i} g_{3 i}}-D \frac{S_{i} G_{2 i}}{S_{i} G_{3 i}}+\Delta y_{\mathrm{c}}$,

where

$$
\Delta x_{\mathrm{c}} \doteq x_{\mathrm{c}}-X_{\mathrm{c}}, \quad \Delta y_{\mathrm{c}} \doteq y_{\mathrm{c}}-Y_{\mathrm{c}},
$$

$\mathbf{g}$ is determined by $\beta$ and $\gamma$, and $\mathbf{G}$ is determined by $\mathrm{B}$ and $\Gamma$. Therefore, it can be seen from equation (20) that the displacement of a peak is associated with eight calibration parameters: $d, D, \Delta x_{\mathrm{c}}, \Delta y_{\mathrm{c}}, \beta, \mathrm{B}, \gamma$ and $\Gamma$.

The $\mathbf{S}$ and $\mathbf{S}$ parameters of equation (20) are determined by the reference and current lattice matrices, respectively; we are actually concerned with eight degrees of freedom for each lattice matrix since isotropic dilation of the lattice matrix does not change the peak positions. Without loss of generality, the $x x$ component of the lattice matrix is set to be a constant value, say 1 , while treating the rest of the components as unknowns. Then, we have 16 unknowns coming from the reference and current lattice matrices, along with the eight unknown calibration parameters mentioned in the last paragraph to be determined.

Now the question is, is it possible to determine the 16 lattice matrix components and eight calibration parameters with 12 spot displacements? The answer is, according to the implicit function theorem (Nocedal \& Wright, 1999, pp. 575-591), as long as the Jacobian matrix of equation (20) (denoted as $\mathbf{J}$ hereinafter) has full rank, at least 12 spot displacements are adequate to determine the 24 parameters, i.e.

$$
\operatorname{det} \mathbf{J}=\operatorname{det} \frac{\partial\left(\Delta x_{1}, \Delta y_{1}, \Delta x_{2}, \Delta y_{2}, \ldots, \Delta x_{12}, \Delta y_{12}\right)}{\partial \mathcal{P}} \neq 0,
$$

where $\mathcal{P}$ denotes the set of all 24 parameters. To further illustrate how $\mathbf{J}$ determines the uniqueness of the solution of equation (20), let us raise an example in which det $\mathbf{J}=0$. If in the reference and current configurations all parameters except $d$ and $D$ are the same, then for any spot we have from equation (20)

$$
\begin{aligned}
& \frac{\partial \Delta x}{\partial d}=\frac{s_{i} g_{1 i}}{s_{i} g_{3 i}}=\frac{S_{i} G_{1 i}}{S_{i} G_{3 i}}=-\frac{\partial \Delta x}{\partial D}, \\
& \frac{\partial \Delta y}{\partial d}=\frac{s_{i} g_{2 i}}{s_{i} g_{3 i}}=\frac{S_{i} G_{2 i}}{S_{i} G_{3 i}}=-\frac{\partial \Delta y}{\partial D} .
\end{aligned}
$$

Therefore, the column of $\mathbf{J}$ corresponding to the partial derivatives with respect to $d$ is opposite to the column corresponding to the partial derivatives with respect to $D$. In this case, $\operatorname{det} \mathbf{J}=0$, and $\mathcal{P}$ cannot be fully determined purely from the spot displacements. Indeed, under such circumstances, the spot displacement is only related to $d-D$ and no longer depends on $d$ or $D$.

The value of $\operatorname{det} \mathbf{J}$ also serves as an indicator of the robustness against DIC errors. Let us denote the errors in DIC as $\delta \Delta x_{i}, \delta \Delta y_{i}, i=1,2, \ldots, 12$ and the resulting errors in the identification of parameters as $\delta \mathcal{P}_{i}, i=1,2, \ldots, 24$. When the errors in DIC are small, we have

$$
\delta \mathcal{P}_{1} \delta \mathcal{P}_{2} \cdots \delta \mathcal{P}_{24}=\frac{1}{|\operatorname{det} \mathbf{J}|} \delta \Delta x_{1} \delta \Delta y_{1} \cdots \delta \Delta x_{12} \delta \Delta y_{12}
$$

Therefore, it is evident from equation (24) that the larger $|\operatorname{det} \mathbf{J}|$ is, the less impact the errors in DIC will exert on the identification of $\mathcal{P}$.

In practice, more than 12 spot displacements are collected and our problem turns into minimizing an objective function:

$$
\begin{aligned}
\Pi \doteq & \sum_{h k l} W_{x}^{h k l}\left(d \frac{s_{i}^{h k l} g_{1 i}}{s_{i}^{h k l} g_{3 i}}-D \frac{S_{i}^{h k l} G_{1 i}}{S_{i}^{h k l} G_{3 i}}+\Delta x_{\mathrm{c}}-\Delta x_{\mathrm{dic}}^{h k l}\right)^{2} \\
& +\sum_{h k l} W_{y}^{h k l}\left(d \frac{s_{i}^{h k l} g_{2 i}}{s_{i}^{h k l} g_{3 i}}-D \frac{S_{i}^{h k l} G_{2 i}}{S_{i}^{h k l} G_{3 i}}+\Delta y_{\mathrm{c}}-\Delta y_{\mathrm{dic}}^{h k l}\right)^{2},
\end{aligned}
$$


where the superscript $h k l$ represents the index of the spot, $\Delta x_{\text {dic }}^{h k l}$ and $\Delta y_{\text {dic }}^{h k l}$ are the displacements of the spot obtained by DIC, and $W_{x}^{h k l}$ and $W_{y}^{h k l}$ are their weights. In practice, to avoid trigonometric calculations, $\beta, \mathrm{B}, \gamma$ and $\Gamma$ are not optimized directly but $t_{\beta / 2} \doteq \tan \beta / 2, t_{\mathrm{B} / 2} \doteq \tan \mathrm{B} / 2, t_{\gamma / 2} \doteq \tan \gamma / 2$ and $t_{\Gamma / 2} \doteq \tan \Gamma / 2$ are optimized instead. Then equation (25) becomes

$$
\begin{aligned}
\Pi= & \sum_{h k l} W_{x}^{h k l}\left(d \frac{s_{i}^{h k l} \tilde{g}_{1 i}}{s_{i}^{h k l} \tilde{g}_{3 i}}-D \frac{S_{i}^{h k l} \tilde{G}_{1 i}}{S_{i}^{h k l} \tilde{G}_{3 i}}+\Delta x_{\mathrm{c}}-\Delta x_{\mathrm{dic}}^{h k l}\right)^{2} \\
& +\sum_{h k l} W_{y}^{h k l}\left(d \frac{s_{i}^{h k l} \tilde{g}_{2 i}}{s_{i}^{h k l} \tilde{g}_{3 i}}-D \frac{S_{i}^{h k l} \tilde{G}_{2 i}}{S_{i}^{h k l} \tilde{G}_{3 i}}+\Delta y_{\mathrm{c}}-\Delta y_{\mathrm{dic}}^{h k l}\right)^{2}
\end{aligned}
$$

where

$$
\begin{gathered}
\tilde{\mathbf{g}} \doteq\left(1+t_{\beta / 2}^{2}\right)\left(1+t_{\gamma / 2}^{2}\right) \mathbf{g}, \\
\tilde{\mathbf{G}} \doteq\left(1+t_{\mathrm{B} / 2}^{2}\right)\left(1+t_{\Gamma / 2}^{2}\right) \mathbf{G} .
\end{gathered}
$$

$\mathcal{P}$ can be obtained by minimizing equation (26) with the spot displacements as the sole input. In summary, the procedure of enhanced Laue-DIC mainly consists of four steps (see Fig. 3):

(1) Take two diffraction images from different positions of a sample. Use, for example, the standard method to index each spot and obtain an estimation of their lattice matrices and calibration parameters.

(2) Measure the displacement of each spot between the two configurations by DIC.

(3) Minimize the discrepancy between the measured displacements and simulated displacements by manipulating the lattice matrices and calibration parameters of the two configurations.

(4) Obtain the orientations and deviatoric elastic strains from the lattice matrices.

The flowchart of enhanced Laue-DIC is given in Fig. 3. The enhanced Laue-DIC method shares some similarities with the method proposed by Maurice et al. (2011) for finding calibration parameters of the high-resolution EBSD setup.

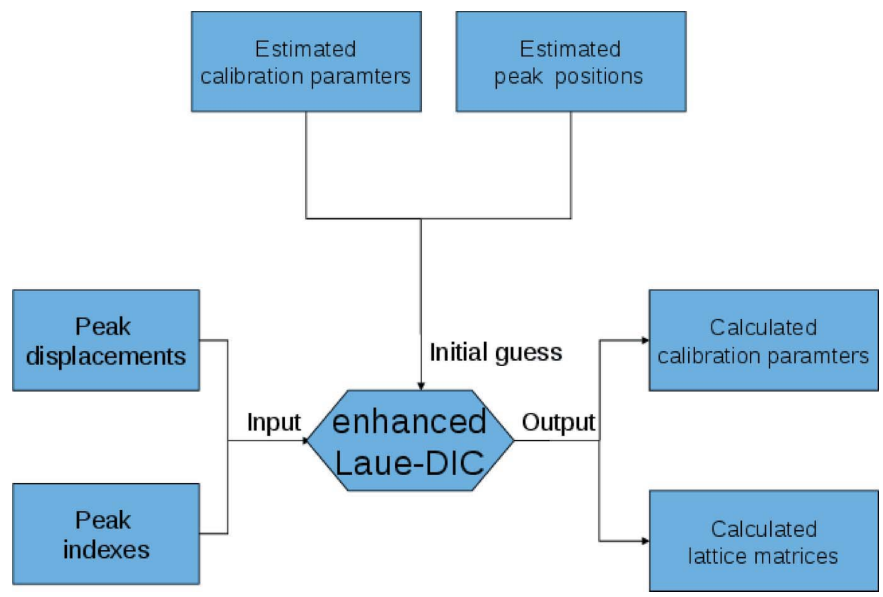

Figure 3

Flowchart of enhanced Laue-DIC.
Table 2

Deviations of calibration parameters, set to be equal to the values of the uncertainties evaluated by Poshadel et al. (2012).

\begin{tabular}{lllll}
\hline$\sigma_{d, D}$ & $\sigma_{x_{c}, X_{c}}$ & $\sigma_{y_{\mathrm{c}} Y_{\mathrm{c}}}$ & $\sigma_{\beta, \mathrm{B}}$ & $\sigma_{\gamma, \Gamma}$ \\
\hline $0.004 \mathrm{~mm}$ & $4.96 \mu \mathrm{m}(0.16$ pixel $)$ & $8.06 \mu \mathrm{m}(0.26$ pixel $)$ & $0.005^{\circ}$ & $0.005^{\circ}$ \\
\hline
\end{tabular}

\section{Numerical test}

Before dealing with experimental data, let us first run some numerical tests to study the performance of different optimization algorithms. The candidate algorithms include the Powell (1964) and L-BFGS-B (Nocedal \& Wright, 1999, pp. 222-249) algorithms. Powell's method, which requires no calculation of the gradient of $\Pi$, is based on line (or onedimensional) minimizations along optimized conjugate directions determined from a local quadratic fit of $\Pi$; these directions are updated after each iteration. The BFGS method is also based on a local quadratic approximation of function $\Pi$, but, unlike Powell's method, it requires the computation of the function gradient. A (quasi)-Newton method is then used for line minimization, with a Hessian matrix not calculated explicitly but estimated from previous evaluation of the function. Complete expressions for the partial derivatives of $\Pi$ are given by Zhang (2015). The number of spots considered in our tests is 40 . The procedure of the numerical tests is given as follows:

(1) Given the exact values of lattice matrices and calibration parameters of two configurations, calculate the theoretical peak positions on the area detector and subsequently their displacements. The considered deformation gradient between the two configurations is taken from a typical experimental data set:

$$
\left[\begin{array}{ccc}
1 & 3.33 \times 10^{-4} & -3.55 \times 10^{-4} \\
9.15 \times 10^{-6} & 1 & -4.49 \times 10^{-4} \\
-3.36 \times 10^{-4} & 2.42 \times 10^{-4} & 1
\end{array}\right]
$$

(2) Slightly deviate the calibration parameters and the simulated peak positions from their exact values according to a normal distribution. According to Poshadel et al. (2012), the assumed error of fitting a peak position is 0.1 pixel; the considered deviations of the calibration parameters are given in Table 2. Note that $y_{\mathrm{c}}$ exhibits a larger uncertainty than $x_{\mathrm{c}}$ because the penetration depth along the $y$ axis adds to the uncertainty (see Fig. 1).

(3) Calculate an estimation of the lattice matrices of the two configurations with the deviated peak positions and deviated calibration parameters by using, for example, the standard method (Chung \& Ice, 1999).

(4) Slightly deviate the theoretical peak displacements from their exact values according to a normal distribution. The deviation of the peak displacements is set to 0.01 pixels in order to mimic the uncertainty of DIC (Bornert et al., 2009; Amiot et al., 2013; Zhang, 2015). 
Table 3

Performance of each individual algorithm.

\begin{tabular}{lllllllllll}
\hline Algorithm & $\bar{e}_{l}$ & $\bar{e}_{L}$ & $\bar{e}_{d}(\mathrm{~mm})$ & $\bar{e}_{D}(\mathrm{~mm})$ & $\bar{e}_{\Delta x_{\mathrm{c}}}(\mathrm{pixel})$ & $\bar{e}_{\Delta y_{\mathrm{c}}}(\mathrm{pixel})$ & $\bar{e}_{\beta}\left({ }^{\circ}\right)$ & $\bar{e}_{\mathrm{B}}\left({ }^{\circ}\right)$ & $\bar{e}_{\gamma}\left({ }^{\circ}\right)$ & $e_{\Gamma}\left({ }^{\circ}\right)$ \\
\hline L-BFGS-B & $5.4 \times 10^{-5}$ & $6.0 \times 10^{-5}$ & $5.3 \times 10^{-2}$ & $1.4 \times 10^{-2}$ & 1.3 & 0.2 & $4.2 \times 10^{-3}$ & $2.8 \times 10^{-3}$ & $6.5 \times 10^{-3}$ & $6.3 \times 10^{-3}$ \\
Powell & $9.6 \times 10^{-5}$ & $9.6 \times 10^{-5}$ & $1.2 \times 10^{-2}$ & $7.5 \times 10^{-2}$ & $4.4 \times 10^{-3}$ & $2.3 \times 10^{-2}$ & $6.7 \times 10^{-4}$ & $4.6 \times 10^{-3}$ & $6.5 \times 10^{-3}$ & $7.5 \times 10^{-3}$ \\
Partial optimization & $3.5 \times 10^{-5}$ & $3.4 \times 10^{-5}$ & $4.0 \times 10^{-4}$ & $3.9 \times 10^{-4}$ & $1.1 \times 10^{-3}$ & $4.4 \times 10^{-2}$ & $4.8 \times 10^{-4}$ & $2.5 \times 10^{-4}$ & $4.8 \times 10^{-4}$ & $4.8 \times 10^{-4}$ \\
\hline
\end{tabular}

(5) Run the optimization to investigate whether and to what accuracy the values of the lattice matrices and calibration parameters can be recovered using the deviated displacements simulated in step (4) as input and the deviated parameters obtained in steps (2) and (3) as initial guess.

For calibration parameters, it is straightforward to define their errors by the discrepancies between the calculated values and the exact ones:

$$
e_{x} \doteq\left|x^{\mathrm{cal}}-x^{\text {exa }}\right|, \quad x \in\left\{d, D, \Delta x_{\mathrm{c}}, \Delta y_{\mathrm{c}}, \beta, \mathrm{B}, \gamma, \Gamma\right\},
$$

where the superscript cal represents values calculated after the minimization process, and the superscript exa represents the exact values. As for the lattice matrices $\mathbf{I}$ and $\mathbf{L}$, the following steps are used to measure their errors:

(1) Divide each component of the lattice matrix by the cube root of the determinant of the lattice matrix in order to exclude the influence of the volume of the unit lattice, which is out of reach, i.e.

$$
\hat{\mathbf{l}} \doteq \frac{\mathbf{l}}{(\operatorname{det} \mathbf{l})^{1 / 3}}, \quad \hat{\mathbf{L}} \doteq \frac{\mathbf{L}}{(\operatorname{det} \mathbf{L})^{1 / 3}},
$$

(2) The errors on $\mathbf{I}$ and $\mathbf{L}$ are defined as

$$
\begin{gathered}
e_{l} \doteq \frac{\sum_{i=1}^{3} \sum_{j=1}^{3}\left|\hat{l}_{i j}^{\mathrm{cal}}-\hat{l}_{i j}^{\mathrm{exa}}\right|}{9}, \\
e_{L} \doteq \frac{\sum_{i=1}^{3} \sum_{j=1}^{3}\left|\hat{L}_{i j}^{\mathrm{cal}}-\hat{L}_{i j}^{\mathrm{exa}}\right|}{9} .
\end{gathered}
$$

Because our numerical test uses randomly generated data, we optimize 500 random cases for each algorithm to make the results statistically significant. The statistical performance of each algorithm is evaluated by the means of $e_{x}$, $x \in\left\{l, L, d, D, \Delta x_{\mathrm{c}}, \Delta y_{\mathrm{c}}, \beta, \mathrm{B}, \gamma, \Gamma\right\}$ of all random cases, denoted as $\bar{e}_{x}$.

The performance of optimization is affected by the iteration count. Fig. 4 depicts the convergence of the L-BFGS-B algorithm, where the $x$ axis represents the iteration counts and the $y$ axis represents the ratio of the cost function $\Pi$ to its initial value $\Pi_{0}$. As can be seen, the value of $\Pi$ drops only slowly when the iteration counts exceed 20 (a similar behavior has been observed when using the Powell method instead of L-BFGS-B). Therefore, a stopping criterion is set for each algorithm employed: the optimization procedure is stopped once the decrease of $\Pi$ after one iteration is smaller than $5 \%$ of the current value of $\Pi$.

The first two rows of Table 3 report the performance of the mentioned algorithms. For both algorithms, the accuracy of the lattice matrices attains the order of $10^{-5}$. However, it is found that the mean errors on calibration parameters are generally larger than the imposed errors tabulated in Table 2. This prompts us to believe that the adopted algorithms are less efficient with calibration parameters than with lattice matrices.

Here, the problem is solved by resorting to partial optimization: all parameters $\mathcal{P}$ are firstly subdivided into lattice matrices, $\mathcal{L}=\left\{\mathbf{I}^{*}, \mathbf{L}^{*}\right\}$, and calibration parameters, denoted as $\mathcal{C}=\left\{d, D, \Delta x_{\mathrm{c}}, \Delta y_{\mathrm{c}}, \beta, \mathrm{B}, \gamma, \Gamma\right\}$. Because $\mathcal{L}$ bears a larger degree of uncertainty than $\mathcal{C}$ (the uncertainty in lattice matrices comes from uncertainties in calibration parameters plus those in peak positions), $\mathcal{L}$ is first optimized with $\mathcal{C}$ fixed, and once the optimization of $\mathcal{L}$ has been finished we go on to optimize $\mathcal{C}$ while keeping $\mathcal{L}$ fixed. This process is stopped once the decrease of $\Pi$ after one iteration is below $5 \%$ of the current value of $\Pi$.

For each stage of partial optimization, i.e. optimizing $\mathcal{L}$ or $\mathcal{C}$, both L-BFGS-B and Powell algorithms were tried, and it is found that the combination of optimizing $\mathcal{L}$ by L-BFGS-B and optimizing $\mathcal{C}$ by Powell gives the most satisfactory results (see the last line of Table 3 ). Comparing the last line with the first two lines, it can be seen that the errors of lattice matrices obtained by partial optimization are of the same magnitude as those obtained by the L-BFGS-B or Powell algorithm, but the errors of the calibration parameters by partial optimization are smaller by an order of $10^{-2}-10^{-1}$. Note that, while partial optimization is so far the best optimization scheme that we have tried, we hope to find in the future an optimization scheme with better performance.

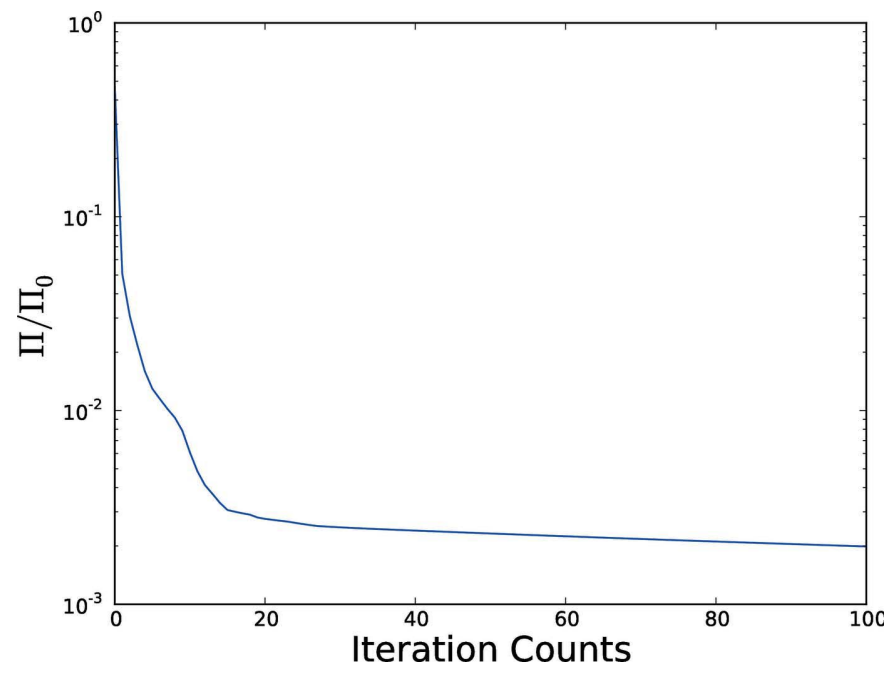

Figure 4

$\Pi / \Pi_{0}$ versus iteration counts. 


\section{Experimental validation}

Now we will use the standard method, original Laue-DIC and enhanced Laue-DIC to treat diffraction images collected from an in situ four-point bending test carried out at beamline BM32 at the ESRF. In this test, an Si monocrystal was mounted onto a four-point bending machine and the bending moment was applied by the four pins holding the monocrystal (see Fig. 5). The size of the crystal was $34.95 \times 7.97 \times$ $2.42 \mathrm{~mm}$. The [100], [010] and [001] crystallographic directions were aligned along the edges of the sample so that the $y$ cross section coincided with the mirror plane of the sample's lattice. This orientation reduces the shear deformation in the $y$ cross section to zero from a theoretical point of view.

The bending moment was applied progressively. At the loadings of 3.9, 88.6 and $199.4 \mathrm{~N}$, the sample was scanned along the $x$ axis of the sample coordinate system with a step size of $8.7 \mu \mathrm{m}$ (see Fig. 5) while maintaining the loading and experimental setup. In such loadings, the corresponding maximum $\sigma_{y y}$ in the scanning line are 2.14, 48.39 and 108.95 MPa, respectively, according to the elastic beam theory. The detector used in our experiment was a VHR X-ray CCD camera whose pixel size and dynamic range were $31 \mu \mathrm{m}$ and 12 bit, respectively. The exposure time for each image was $0.2 \mathrm{~s}$. The obtained Laue spots were more or less streaked by dynamic effects (Yan \& Noyan, 2006) (see Fig. 6). Prior to scanning each sample, a diffraction image was obtained from a Ge monocrystal positioned on the surface of the sample to determine the calibration parameters of the setup (Robach et al., 2011).

In terms of DIC setting, the shape function was set to be a zero-order polynomial, i.e. the motion of the spot on the detector screen was assumed to be pure translation (the shapes of the spots in two diffraction images show a high resemblance). This resemblance could be a posteriori confirmed by the value of the correlation coefficient, the quantification of the resemblance of the images, which turned out to be of the order of $1 \times 10^{-3}$ [the value of the correlation coefficient ranges from 0 to 2; a smaller value means a higher resemblance; when it becomes zero, the two images are identical; see equation (4)]. The weights in equation (26) were designated to be

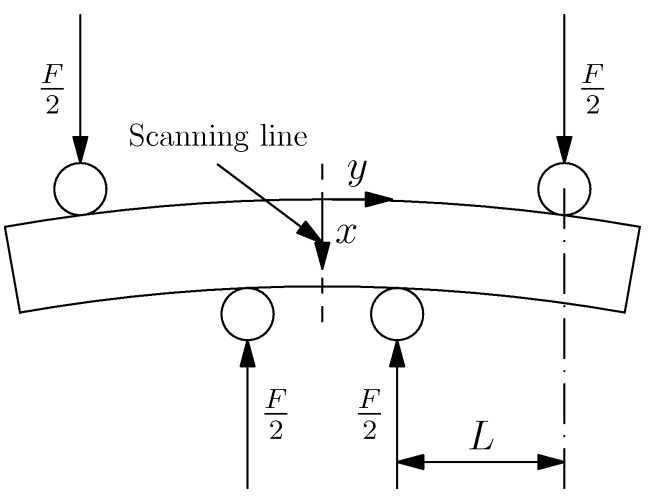

Figure 5

The in situ test carried out at beamline BM32 at the ESRF. $L=8.5 \mathrm{~mm}$.

$$
W_{x}^{h k l}=W_{y}^{h k l}=1-C^{h k l},
$$

where $C^{h k l}$ is the correlation coefficient of the spot with index $(h k l)$. A higher resemblance usually means more credibility in the measurement of the displacements.

An analytical solution of stress based on anisotropic elastic theory (Rand \& Rovenski, 2005) was used to provide a reference to the measurements. For homogeneous anisotropic material, the analytical solution of stress is independent of the orientation and elastic constants, as long as classical beam theory holds true (i.e. for a sufficiently large aspect ratio of the beam), which is assumed here:

$$
\left\{\begin{array}{l}
\sigma_{y y}^{\prime}=-\frac{2}{3} \frac{M}{I_{z}}\left(x-\frac{X}{2}\right), \\
\sigma_{x x}^{\prime}=\sigma_{z z}^{\prime}=\frac{1}{3} \frac{M}{I_{z}}\left(x-\frac{X}{2}\right), \\
\tau_{x y}=\tau_{y z}=\tau_{z x}=0,
\end{array}\right.
$$

where $\sigma^{\prime}$ and $\tau$ represent the deviatoric stress components, $M$ is the bending moment calculated by $M=(F / 2) L$ from Fig. 5 , $X$ is the sample size in the $x$ direction of the sample coordinate system (see Fig. 5), $I_{z}=Z X^{3} / 12$ is the area moment of inertia of the $y$ cross section, and $Z$ is the size of the sample in the $z$ direction. The elastic constants for the $\mathrm{Si}$ monocrystal are $C_{11}=166 \mathrm{GPa}, C_{12}=64 \mathrm{GPa}$ and $C_{44}=79 \mathrm{GPa}$ (Keating, 1966).

Although with equation (33) the theoretical stress profiles could be calculated with the force measured by the sensor (nominal force), these reference stress profiles might be prone to error owing to the uncertainties in the measured force, the sample sizes and the positioning of the pins. We would rather apply a linear regression to the measured stress profiles to obtain reference stress profiles, since it is indicated from equation (33) that the stress should be linearly distributed along the scanning line.

\subsection{Comparison between the standard method and enhanced Laue-DIC}

The sequences of diffraction images were processed with the standard method and enhanced Laue-DIC, and we compare their results. First, we used the standard method to obtain the lattice matrix and calibration parameters for each Laue image. Then, all illumination sites were grouped into pairs, each pair containing two adjacent illumination sites (see

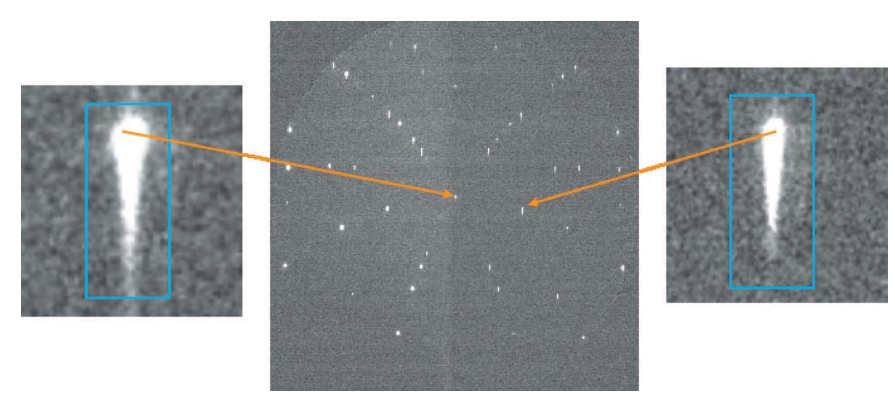

Figure 6

The streaked spots in a diffraction image of $\mathrm{Si}$. 
Table 4

RMS of discrepancies between the measurements and the fitted solution of the $\sigma_{y y}^{\prime}$ profiles (unit: MPa).

\begin{tabular}{llll}
\hline & $3.9 \mathrm{~N}$ & $88.6 \mathrm{~N}$ & $199.4 \mathrm{~N}$ \\
\hline Standard method & 1.94 & 6.50 & 3.23 \\
Enhanced Laue-DIC & 2.17 & 3.95 & 2.10 \\
\hline
\end{tabular}

Fig. 7). Pairing the images in this way maximizes the resemblance between them and is therefore beneficial for DIC. Finally, we applied DIC to each pair and used the aforementioned procedure of enhanced Laue-DIC (see Fig. 3) to refine lattice matrices and calibration parameters at each illumination site. In each pair of images, $\sim 30$ spots were considered.

The profiles of $\sigma_{y y}^{\prime}$ along the $x$ axis at 3.9, 88.6 and $199.4 \mathrm{~N}$ with both the standard method and enhanced Laue-DIC are plotted in Fig. 8. It appears that the measurements by enhanced Laue-DIC exhibit less fluctuation around the solutions value. A short span of the $88.6 \mathrm{~N}$ stress profile next to the neutral fiber at $x \simeq 1 \mathrm{~mm}$ can be also observed, departing from the expected linear trend. This anomaly, which does not happen for other loading stages, is unfortunately not explained; it probably comes from some drift in the experimental conditions. It is not due to the data processing as it can be observed with the three methods tested here, namely standard, Laue-DIC and enhanced Laue-DIC.

A more quantitative evaluation lies in comparing the root mean square (RMS) of the discrepancies between the measurements and the fitted solution:

$$
\mathrm{RMS} \doteq\left\{\frac{\sum_{i=1}^{n}\left[\sigma_{(i)}^{\text {meas }}-\sigma_{(i)}^{\mathrm{fit}}\right]^{2}}{n}\right\}^{1 / 2}
$$

The RMS values of $\sigma_{y y}^{\prime}$ for the standard and enhanced LaueDIC methods are given in Table 4. The results show that at loadings of 88.6 and $199.4 \mathrm{~N}$ enhanced Laue-DIC can reduce the discrepancies between the measurements and the fitted solution by the order of 1-3 MPa. But at the loading of $3.9 \mathrm{~N}$, the discrepancy of enhanced Laue-DIC is slightly larger; this may be because at such loading the peak displacements are very small and not precisely captured by DIC. Indeed, the accuracy of DIC for almost null displacements has been observed to be worse than for finite displacements (Amiot et al., 2013).

Table 5 gives the fitted slopes of the $\sigma_{y y}^{\prime}$ profiles and the nominal slopes calculated from equation (33). It can be seen that for the loads of 3.9 and $88.6 \mathrm{~N}$ the nominal slope and the fitted slope differ only slightly; for the load of $199.4 \mathrm{~N}$ the nominal slope and the fitted slope differ more. In any case,

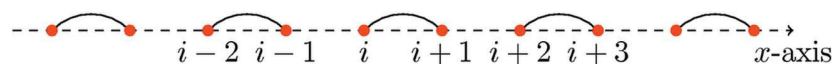

Figure 7

Correlation pairs: red spots represent illumination sites, black curves represent correlations between two spots and the dashed line represents the scanning line $x$ (see Fig. 1).
Table 5

Fitted and nominal slopes of $\sigma_{y y}^{\prime}$ profiles (unit: $\mathrm{MPa} \mathrm{mm} \mathrm{m}^{-1}$ ).

\begin{tabular}{lccc}
\hline & $3.9 \mathrm{~N}$ & $88.6 \mathrm{~N}$ & $199.4 \mathrm{~N}$ \\
\hline Fitted slope from standard method & -1.99 & -24.59 & -54.50 \\
Fitted slope from enhanced Laue-DIC & -1.67 & -26.10 & -55.23 \\
Nominal slope & -1.18 & -26.66 & -60.03 \\
\hline
\end{tabular}

enhanced Laue-DIC provides an evaluation closer to the nominal values.

Other components of deviatoric stresses are now investigated. For brevity, only the results for $199.4 \mathrm{~N}$ are given (see Fig. 9). It is observed that enhanced Laue-DIC always reduces the fluctuations of profiles. Nevertheless, we observe at both ends of the scanning line abnormal trends deviating from the supposed linearity of the stress profiles in equation (33). Such trends are also observed in the $\sigma_{y y}^{\prime}$ profiles, but with a much lower, almost negligible, amplitude. However, these deviations should not be due to enhanced Laue-DIC itself, because they also exist in the results of the standard method. Some reasons

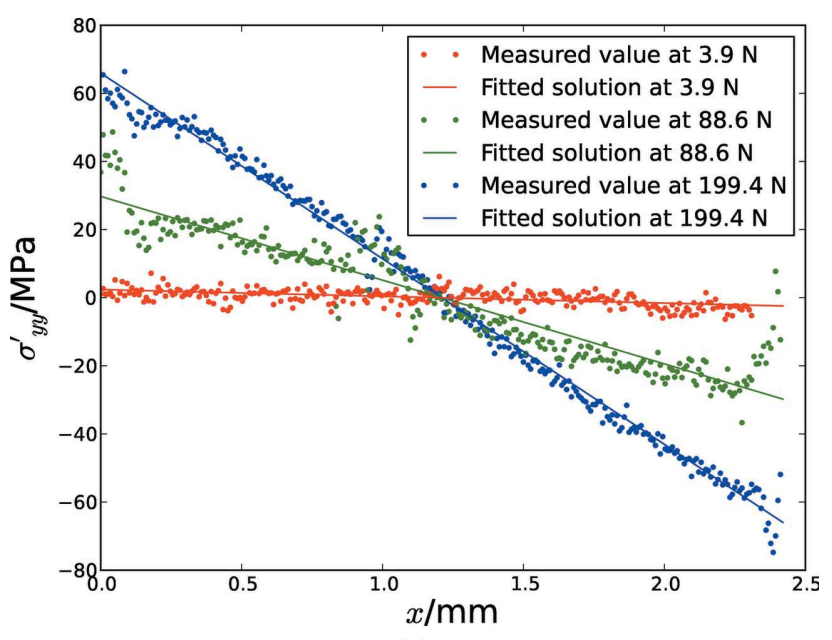

(a)

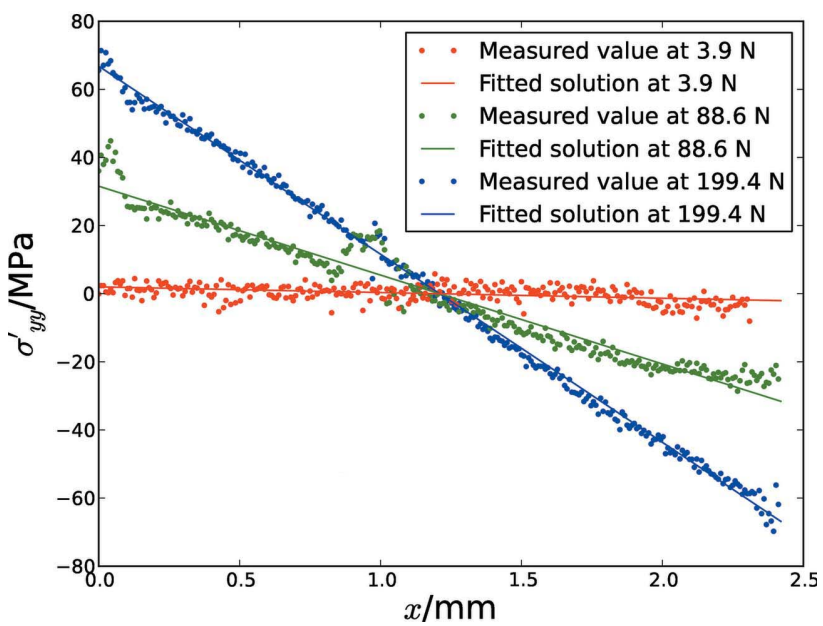

(b)

Figure 8

Comparison of the measured $\sigma_{y y}^{\prime}$ profile by $(a)$ the standard method and (b) enhanced Laue-DIC. 
other than data treatment that may account for these abnormalities include the following:

(1) The analytical solution gives the stress on the specimen surface, while measurements give the stress somewhere beneath the surface owing to the penetration of the X-rays. In addition, the measurement provides a kind of averaged stress over the area irradiated by the beam, which encompasses a (small) range of the scanning line, because of the tilt angle between the beam and the sample.

(2) The sample might not be under pure bending conditions, because of the imperfections of the setup and of the finite aspect ratio of the sample. In addition, the polished surface of the sample might not be perfectly flat.

Aside from the stress profile, enhanced Laue-DIC also allows for the evaluation of the calibration parameters of each illumination site. As mentioned before, a piece of Ge monocrystal was glued onto the sample surface to get a first evaluation of the calibration parameters before scanning. We call the obtained calibration parameters the nominal ones and

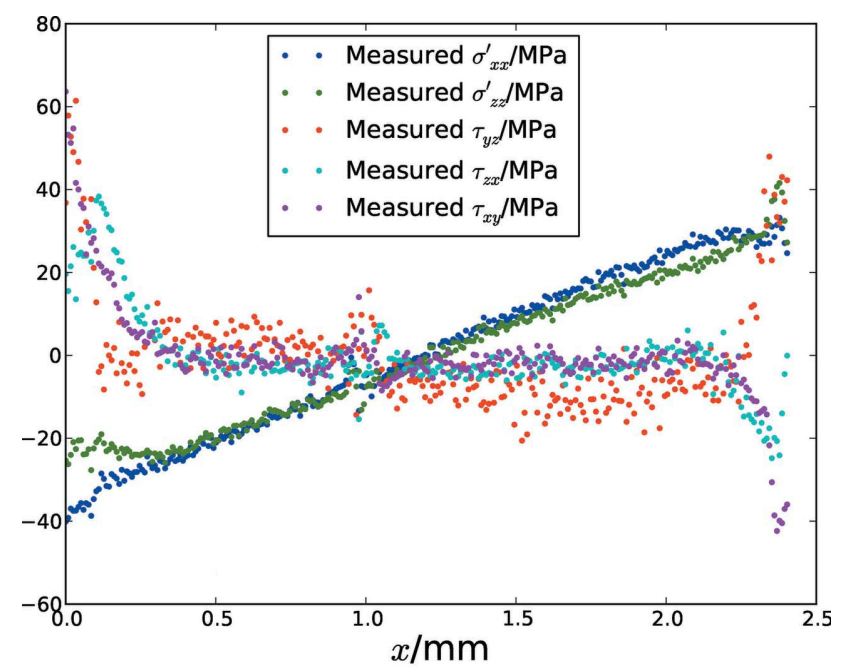

(a)

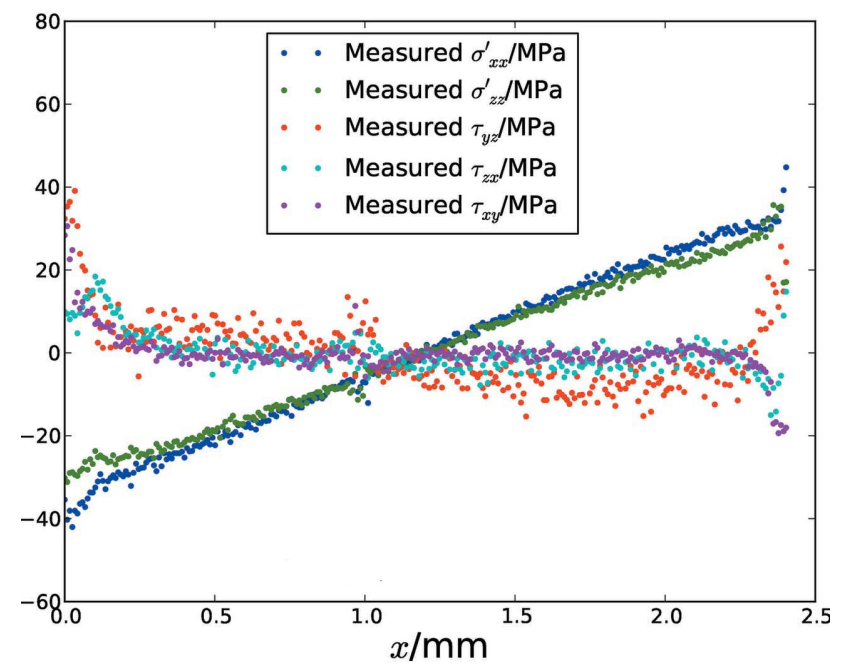

(b)

Figure 9

Comparison of stress profiles of other components by $(a)$ the standard method and $(b)$ enhanced Laue-DIC.
Table 6

Mean biases and fluctuations of calibration parameters (pixel size: $31 \mu \mathrm{m})$.

\begin{tabular}{llllll}
\hline & $d(\mathrm{~mm})$ & $\Delta x_{\mathrm{c}}(\mathrm{pix})$ & $\Delta y_{\mathrm{c}}(\mathrm{pix})$ & $\beta\left(^{\circ}\right)$ & $\gamma\left({ }^{\circ}\right)$ \\
\hline $\bar{e}$ & $2.8 \times 10^{-3}$ & $1.3 \times 10^{-3}$ & $4.6 \times 10^{-3}$ & $7.7 \times 10^{-4}$ & $2.6 \times 10^{-3}$ \\
$\sigma$ & $4.9 \times 10^{-3}$ & $9.1 \times 10^{-3}$ & $1.5 \times 10^{-2}$ & $3.3 \times 10^{-3}$ & $4.2 \times 10^{-3}$ \\
\hline
\end{tabular}

denote them by a superscript nom. Then, the deviation of the calibration parameters was quantified by the bias of the mean $x^{\mathrm{cal}}$ from the nominal value, e.g. $\bar{e}_{x}=\left|\bar{x}^{\mathrm{cal}}-x^{\mathrm{nom}}\right|$, and the fluctuation $\sigma_{x}$, the standard deviation of $x^{\text {cal }}$, in which $x \in\left\{d, \Delta x_{\mathrm{c}}, \Delta y_{\mathrm{c}}, \beta, \gamma\right\}$ (we do not use capital letters $D, \mathrm{~B}$ and $\Gamma$ any more because we do not have to distinguish the reference and current configuration here). Note that $\Delta x_{\mathrm{c}}^{\text {nom }} \equiv 0$ and $\Delta y_{\mathrm{c}}^{\text {nom }} \equiv 0$ as a consequence of the definition of these parameters (the nominal values of $\Delta x_{\mathrm{c}}^{\text {nom }}$ and $\Delta y_{\mathrm{c}}^{\text {nom }}$ are the same for all illumination sites). The results of biases and fluctuations are tabulated in Table 6.

From Table 6, it can be seen that the fluctuations of the calibration parameters are larger than their corresponding mean deviations. In comparison with Table 2, the calculated fluctuations of the calibration parameters are of a similar magnitude to those reported by Poshadel et al. (2012) for $d, \beta$ and $\gamma$. The fluctuations of $\Delta x_{\mathrm{c}}$ and $\Delta y_{\mathrm{c}}$ are smaller than those reported for $x_{\mathrm{c}}$ and $y_{\mathrm{c}}$, consistent with the fact that in enhanced Laue DIC one does not seek the exact absolute position of the normal projection of the illuminated region on the detector, but only its possible deviations between compared configurations.

\subsection{Comparison between original Laue-DIC and enhanced Laue-DIC}

With the same sequence of diffraction images, we can also run both original and enhanced Laue-DIC analysis, and compare the stress increments from original Laue-DIC with those from enhanced Laue-DIC. Here, we use a different pairing scheme from the one in the previous section: the lattice in the middle of the scanning line (step number N/2) is chosen as the reference one, and we perform both original and enhanced Laue-DIC with the other lattices in the scanning line (see Fig. 10) to calculate the stress increment with respect to the reference lattice, i.e. $\Delta \sigma_{i j} \doteq \sigma_{i j}-\sigma_{i j}^{\text {ref }}, i, j \in\{x, y, z\}$. This image-pairing scheme is adapted for Laue-DIC, for which the used reference image should correspond to a well known lattice. Here, point $N / 2$ lies in the beam neutral fiber and is therefore assumed to be stress free. In addition, when used for

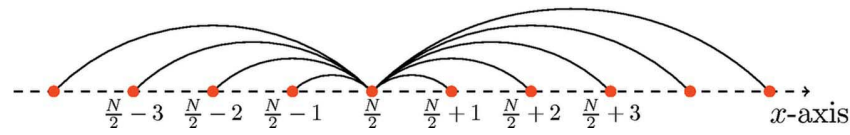

Figure 10

Correlation pairs: red spots represent illumination sites, black curves represent correlations between two spots and the dashed line represents the scanning line $x$ (see Fig. 1). $N$ is the total number of illumination sites. 
the enhanced Laue-DIC method, this scheme allows evaluation of the stress and calibration parameters many $(=N-1)$ times for the same reference configuration and hence allows estimation of the errors on the calibration and reference lattice at that point.

Fig. 11 plots the profiles of $\Delta \sigma_{y y}^{\prime}$ at 3.9, 88.6 and $199.4 \mathrm{~N}$ with both original and enhanced Laue-DIC. The RMS values of the discrepancies between the measurements and the fitted solution are given in Table 7 . Both qualitative and quantitative comparison of the $\Delta \sigma_{y y}^{\prime}$ profiles by original and enhanced Laue-DIC show that the performance of the two methods is very similar.

Fig. 12 plots the profiles of the increments of the other stress components at the loading of $199.4 \mathrm{~N}$. Again, it is observed that these profiles are very similar to their counterparts. However, the profile of $\Delta \tau_{y z}$ by enhanced Laue-DIC exhibits more fluctuation than its counterpart by original Laue-DIC.

To sum up, the stress profiles obtained by the two versions of Laue-DIC are very similar, but the profiles by enhanced Laue-DIC appear slightly more fluctuated. We are still not certain about the cause of this phenomenon; either enhanced

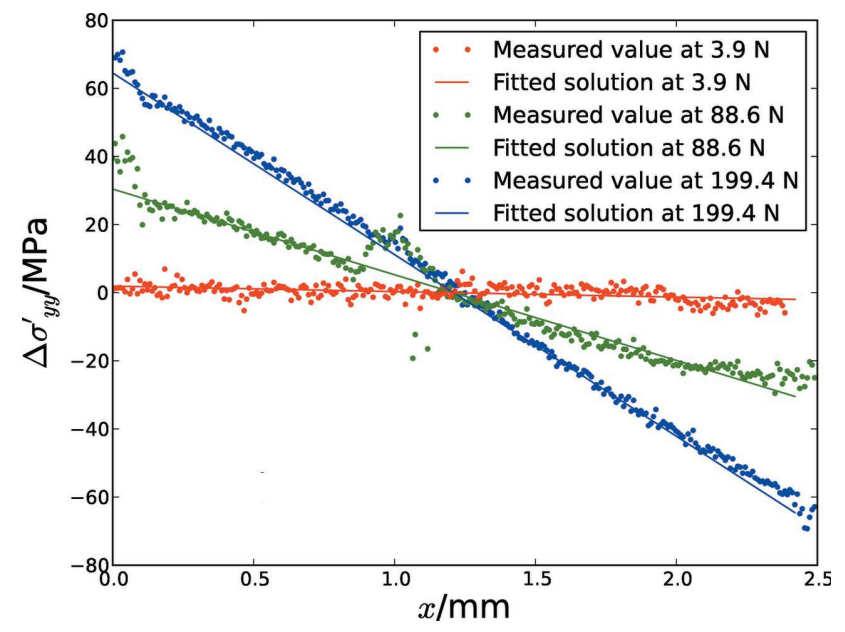

(a)

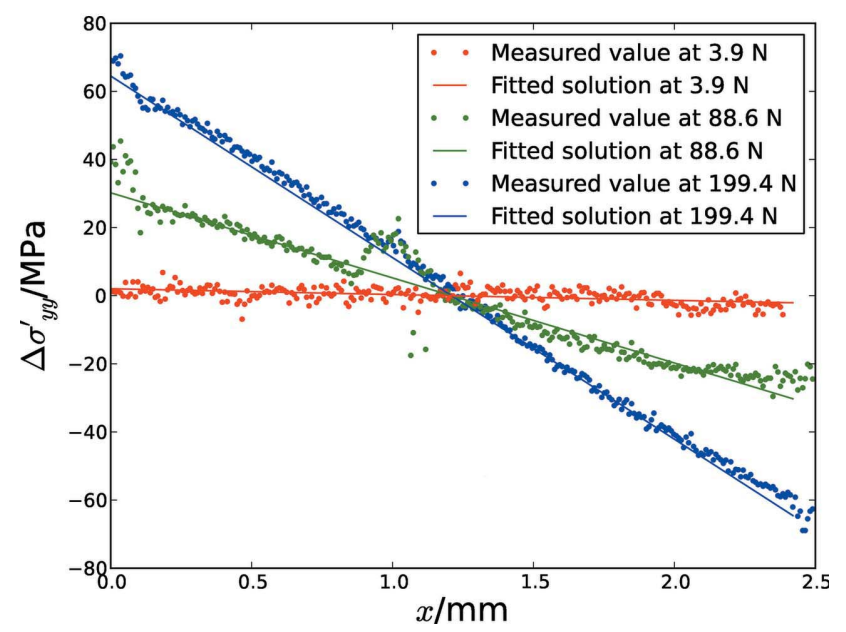

(b)

Figure 11

Comparison of the measured $\Delta \sigma_{y y}^{\prime}$ profile by (a) original Laue-DIC and (b) enhanced Laue-DIC.
Table 7

RMS of discrepancies between the measurements and the analytical solution (unit: $\mathrm{MPa}$ ).

\begin{tabular}{llll}
\hline & $3.9 \mathrm{~N}$ & $88.6 \mathrm{~N}$ & $199.4 \mathrm{~N}$ \\
\hline Original Laue-DIC & 1.94 & 4.53 & 1.82 \\
Enhanced Laue-DIC & 1.93 & 4.50 & 1.86 \\
\hline
\end{tabular}

Laue-DIC has more accurately reflected the inhomogeneity of stress distribution at micrometre scale, or enhanced Laue-DIC has encountered greater difficulty in its optimization. As already discussed, large uncertainties of the calibration parameters and the reference lattice matrix, together with a good image quality (low noise, high resolution, high peak intensity and large spot numbers), are required to highlight the merits of enhanced Laue-DIC. Otherwise, it is not worthwhile to apply enhanced Laue-DIC to work out 24 unknowns if we could have a fairly reasonable estimation of them. This point will be investigated in detail in a forthcoming paper by means

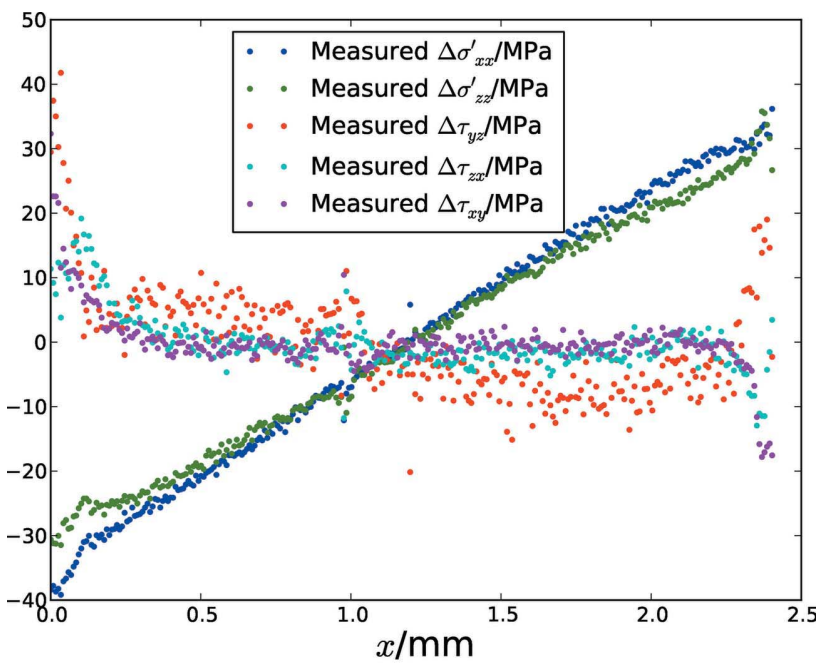

(a)

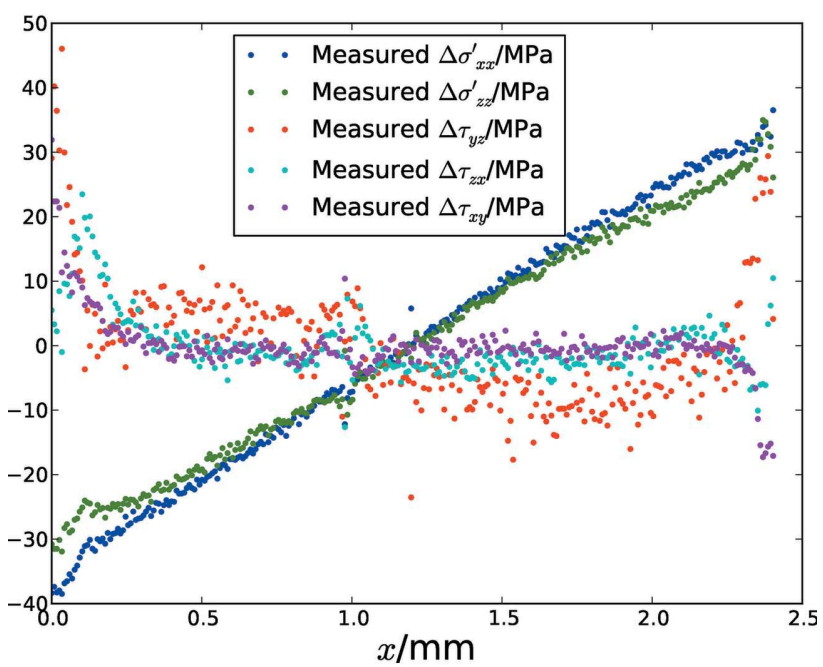

(b)

Figure 12

Comparison of profiles of increments of other stress components by $(a)$ original Laue-DIC and (b) enhanced Laue-DIC. 
of a statistical analysis of randomly distorted numerical Laue images.

As can be seen from Fig. 10, each time enhanced Laue-DIC is performed, the stress of the reference configuration is evaluated. Thereby the stress of the reference illumination site is evaluated $N-1$ times when all pairs of images in Fig. 10 have been analyzed ( $N$ represents the total number of illumination sites, in our case $N=284$ ). The $N-1$ evaluations enable us to calculate the standard deviations of each stress components of the reference configuration: $\sigma_{\sigma_{x x}}=1.43 \mathrm{MPa}$, $\sigma_{\sigma_{y y}}=0.44 \mathrm{MPa}, \sigma_{\sigma_{z z}}=1.55 \mathrm{MPa}, \sigma_{\tau_{y z}}=2.67 \mathrm{MPa}, \sigma_{\tau_{z x}}=$ $2.06 \mathrm{MPa}$ and $\sigma_{\tau_{x y}}=0.56 \mathrm{MPa}$. It is observed that the $\tau_{y z}$ and $\tau_{x z}$ components exhibit larger uncertainties. In the same way, the standard deviations of the calibration parameters at the same position are $\sigma_{d}=4.3 \times 10^{-5} \mathrm{~mm}, \quad \sigma_{\Delta x_{\mathrm{c}}}=3.4 \times$ $10^{-4}$ pixels, $\sigma_{\Delta y_{\mathrm{c}}}=1.3 \times 10^{-3}$ pixels, $\sigma_{\beta}=8.2 \times 10^{-4 \circ}$ and $\sigma_{\gamma}=5.8 \times 10^{-4} \stackrel{\circ}{\circ}$. Unlike the uncertainties tabulated in Table 6 , the uncertainties here are only associated with the errors of the peak displacements and the performance of numerical optimization, because they all refer to the reference configuration.

\section{Summary}

In this work, the so-called enhanced Laue-DIC method is proposed to derive lattice matrices and calibration parameters of two configurations. Compared to the standard method, the main features of the enhanced Laue-DIC method can be summarized as follow:

(1) Enhanced Laue-DIC calculates the lattice matrices and calibration parameters simultaneously, while the standard method calculates them separately.

(2) Enhanced Laue-DIC uses two diffraction images to obtain the lattice matrices and calibration parameters of the two configurations, while the standard method uses only one image to obtain the lattice matrix (and a separate calibrant image taken in advance on a reference specimen for the calibration parameters).

(3) Although enhanced Laue-DIC usually uses lattice matrices and calibration parameters obtained by the standard method as initial guess, it essentially uses spot displacements as the sole source of information while the standard method uses the absolute positions.

Enhanced Laue-DIC differs from original Laue-DIC in two aspects (with the sole assumption that the crystal in its natural unstressed state exhibits cubic symmetry): (i) enhanced LaueDIC calculates the deviatoric elastic strain and orientation of the lattice, while original Laue-DIC calculates the elastic strain increment and relative rotation between two configurations; (ii) enhanced Laue-DIC calculates calibration parameters while original Laue-DIC does not.

We have presented the performance of the standard method, original Laue-DIC and enhanced Laue-DIC by treating a diffraction image sequence of scanning an $\mathrm{Si}$ monocrystalline sample subjected to bending. Compared to the results of the standard method, those of enhanced LaueDIC exhibit less fluctuation. However, in terms of stress increment, the results of original Laue-DIC appear slightly less fluctuated.

Further improvements for the proposed enhanced LaueDIC method may include the following:

(1) Apply this method to samples with a rough surface, as it allows for determination of both lattice matrices and diffraction location and is tolerant to significant evolution of calibration parameters.

(2) As pointed out in equation (24), the determinant of the Jacobian matrix $\operatorname{det} \mathbf{J}$ influences the robustness against errors in DIC measurement. Therefore in a future experiment it would be possible to deliberately manipulate the calibration parameters to increase $\operatorname{det} \mathbf{J}$ and hence the robustness against DIC errors. Also, as the detector position seems to be more easily optimized than its orientation (Zhang, 2015), one could imagine deriving intermediate methods between Laue-DIC and enhanced Laue-DIC in which only part of the calibration parameters would be refined.

(3) As revealed from $\S 4$, the optimization algorithm is vital for the performance of enhanced Laue-DIC. In future, we hope to find a more efficient algorithm, with which the parameters could quickly converge to their accurate values.

\section{Acknowledgements}

We are very grateful to J. P. Vassali (ESRF) for his help on sample preparation, and to O. Robach and J. S Micha (CEA and CNRS) for their help in performing microdiffraction experiments at BM32-ESRF and their very fruitful comments on this work. We also acknowledge financial support provided by the French ANR agency (MICROSTRESS project, No. ANR-11-BS09-030) and the China Scholarship Council.

\section{References}

Amiot, F., Bornert, M., Doumalin, P., Dupré, J. C., Fazzini, M., Orteu, J. J., Poilâne, C., Robert, L., Rotinat, R., Toussaint, E., Wattrisse, B. \& Wienin, J. S. (2013). Strain, 49, 483-496.

Barabash, R., Ice, G. E., Larson, B. C., Pharr, G. M., Chung, K.-S. \& Yang, W. (2001). Appl. Phys. Lett. 79, 749-751.

Bornert, M., Brémand, F., Doumalin, P., Dupré, J.-C., Fazzini, M., Grédiac, M., Hild, F., Mistou, S., Molimard, J., Orteu, J.-J., Robert, L., Surrel, Y., Vacher, P. \& Wattrisse, B. (2009). Exp. Mech. 49, 353370.

Bornert, M., Valès, F., Gharbi, H. \& Nguyen Minh, D. (2010). Strain, 46, 33-46.

Castelnau, O., Drakopoulos, M., Schroer, C., Snigireva, I., Snigirev, A. \& Ungar, T. (2001). Nucl. Instrum. Methods Phys. Res. Sect. A, 467468, 1245-1248.

Chung, J. \& Ice, G. (1999). J. Appl. Phys, 86, 5249-5255.

Daveau, G. (2012). PhD thesis, Ecole Centrale de Paris, ChâtenayMalabry, France.

Doumalin, P. \& Bornert, M. (2000). Interferometry in Speckle Light, pp. 67-74. Berlin, Heidelberg: Springer.

Hofmann, F., Eve, S., Belnoue, J., Micha, J. \& Korsunsky, A. (2011). Nucl. Instrum. Methods Phys. Res. Sect. A, 660, 130-137.

Keating, P. N. (1966). Phys. Rev. 145, 637-645.

Maaß, R., Petegem, S. V., Borca, C. \& Swygenhoven, H. V. (2009). Mater. Sci. Eng. A, 524, 40-45.

Macherauch, E. (1966). Exp. Mech. 6, 140-153. 
Maurice, C., Dzieciol, K. \& Fortunier, R. (2011). Ultramicroscopy, 111, 140-148.

Nocedal, J. \& Wright, S. J. (1999). Numerical Optimization. New York: Springer.

Ohashi, T., Barabash, R., Pang, J., Ice, G. \& Barabash, O. (2009). Int. J. Plast. 25, 920-941.

Peters, W. H. \& Ranson, W. F. (1982). Opt. Eng. 21, 213427.

Petit, J., Castelnau, O., Bornert, M., Zhang, F. G., Hofmann, F., Korsunsky, A. M., Faurie, D., Le Bourlot, C., Micha, J. S., Robach, O. \& Ulrich, O. (2015). J. Synchrotron Rad. 22, 980-994.

Plancher, E., Petit, J., Maurice, C., Favier, V., Saintoyant, L., Loisnard, D., Rupin, N., Marijon, J.-B., Ulrich, O., Bornert, M., Micha, J.-S., Robach, O. \& Castelnau, O. (2015). Exp. Mech. Submitted.

Poshadel, A., Dawson, P. \& Johnson, G. (2012). J. Synchrotron Rad. 19, 237-244.

Powell, M. J. D. (1964). Comput. J. 7, 155-162.

Rand, O. \& Rovenski, V. (2005). Analytical Methods in Anisotropic Elasticity, pp. 215-248. Boston: Birkhäuser.

Robach, O., Kirchlechner, C., Micha, J.-S., Ulrich, O., Biquard, X., Geaymond, O., Castelnau, O., Bornert, M., Petit, J., Berveiller, S., Sicardy, O., Villanova, J. \& Rieutord, F. (2014). Strain and Dislocation Gradients from Diffraction, ch. 5, pp. 156-204. London: Imperial College Press, World Scientific Publishing.

Robach, O., Micha, J.-S., Ulrich, O. \& Gergaud, P. (2011). J. Appl. Cryst. 44, 688-696.
Tamura, N. (2014). Strain and Dislocation Gradients from Diffraction, ch. 4, pp. 125-155. London: Imperial College Press, World Scientific Publishing.

Tamura, N., MacDowell, A. A., Spolenak, R., Valek, B. C., Bravman, J. C., Brown, W. L., Celestre, R. S., Padmore, H. A., Batterman, B. W. \& Patel, J. R. (2003). J. Synchrotron Rad. 10, 137-143.

Tong, W. (2005). Strain, 41, 167-175.

Ulrich, O., Biquard, X., Bleuet, P., Geaymond, O., Gergaud, P., Micha, J. S., Robach, O. \& Rieutord, F. (2011). Rev. Sci. Instrum. 82, 033908.

Villert, S., Maurice, C., Wyon, C. \& Fortunier, R. (2009). J. Microsc. 233, 290-301.

Waltersperger, S., Olieric, V., Pradervand, C., Glettig, W., Salathe, M., Fuchs, M. R., Curtin, A., Wang, X., Ebner, S., Panepucci, E., Weinert, T., Schulze-Briese, C. \& Wang, M. (2015). J. Synchrotron Rad. 22, 895-900.

Wang, Y. Q., Sutton, M. A., Bruck, H. A. \& Schreier, H. W. (2009). Strain, 45, 160-178.

Xu, W., Lauer, K., Chu, Y. \& Nazaretski, E. (2014). J. Synchrotron Rad. 21, 1367-1369.

Yan, H. \& Noyan, I. C. (2006). J. Appl. Cryst. 39, 320-325.

Yang, W. G., Larson, B. C., Tischler, J. Z., Ice, G. E., Budai, J. D. \& Liu, W. (2004). Micron, 35, 431-439.

Zhang, F. G. (2015). PhD thesis, Arts et Métiers ParisTech, Paris, France. 